\title{
Review Article \\ Clinical Aspects of HyFoSy as Tubal Patency Test in Subfertility Workup
}

\author{
Niek Exalto ${ }^{1}{ }^{1}$ and Mark Hans Emanuel ${ }^{2,3}$ \\ ${ }^{1}$ Division of Obstetrics and Prenatal Medicine, Department of Obstetrics and Gynaecology, Erasmus MC, University Medical Centre, \\ Rotterdam, Netherlands \\ ${ }^{2}$ Division of Woman and Baby, Department of Gynaecology and Reproductive Medicine, University Medical Centre, \\ Utrecht, Netherlands \\ ${ }^{3}$ Department of (Uro)gynaecology, University Hospital, Ghent, Belgium
}

Correspondence should be addressed to Niek Exalto; n.exalto@erasmusmc.nl

Received 31 March 2019; Accepted 4 June 2019; Published 8 July 2019

Academic Editor: Alessandro Favilli

Copyright ( 2019 Niek Exalto and Mark Hans Emanuel. This is an open access article distributed under the Creative Commons Attribution License, which permits unrestricted use, distribution, and reproduction in any medium, provided the original work is properly cited.

\begin{abstract}
Background. Tubal patency testing is an essential part of female subfertility evaluation. Traditionally, hysterosalpingography (HSG) was the first step to evaluate tubal patency. However, during the past decade Hysterosalpingo-Contrast Sonography (HyCoSy) was introduced in order to avoid radiation exposure and Hysterosalpingo-Foam Sonography (HyFoSy) has been developed as a safe and less painful alternative. Objectives and Rationale. The aim of this narrative review is to provide an overview of the currently available HyFoSy literature and related clinical aspects. Search Methods. A literature search was conducted using PubMed and Embase from the introduction of HyFoSy to March 2019. Unfortunately, a meta-analysis was not possible due to a too small number of studies, being mutually incomparable for the various subjects of clinical aspects, even for the reliability as a test for tubal patency. Outcomes. Nine small studies concluded that the accuracy and effectiveness as a test for tubal patency of 2D- and 3D-HyFoSy are comparable or even better than HSG or HyCoSy. With or without using Doppler techniques, 3D-HyFoSy does not seem to offer benefits above real-time 2D-HyFoSy. Five studies reported on pain and discomfort during HyFoSy, concluding that HyFoSy is a well-tolerated, less painful procedure compared to HSG, without a need for the use of analgesics. There are suggestions about an increased pregnancy rate in the first three cycles after the procedure but in no studies pregnancy outcome after HyFoSy was compared with other or no intervention. Wider Implications. HyFoSy is a promising and safe alternative for HSG with regard to accuracy and effectiveness. HyFoSy lacks radiation and iodine exposure and is a well-tolerated and less painful procedure than HSG, without the need for analgesics. However more research is needed to make clear statements regarding a therapeutic effect of HyFoSy.
\end{abstract}

\section{Introduction}

Tubal patency testing is an essential part of female subfertility evaluation [1]. Tubal abnormalities are seen in $30-40 \%$ of female subfertility patients [2]. Laparoscopy (LSC) with chromopertubation is considered to be the gold standard diagnostic procedure [3]. LSC is an invasive test, with a risk of intra-abdominal bleeding, visceral damage, and risks related to general anaesthesia. Traditionally, hysterosalpingography (HSG) is a less invasive first-step alternative, which has been used for decades. Considering the disadvantages of HSG such as radiation exposure, discomfort, or even abdominal pain, Hysterosalpingo-Contrast Sonography (HyCoSy) was introduced as an alternative $[4,5]$ using either contrast media or saline. The accuracy of HyCoSy is comparable to that of HSG and LSC $[6,7]$ being reference standards for tubal patency testing. An advantage of ultrasound is that, in addition to tubal patency, information is also obtained about pelvic anatomy including uterus and ovaries. Avoiding pregnancy in the treatment cycle is extremely important in HSG procedures.

There are indications that the accuracy of HyCoSy may improve by using $3 \mathrm{D}$ imaging techniques $[8,9]$. Recently it was claimed in a LSC controlled study [10] that not only tubal 
patency but also peritubal adhesion could be assessed with HyCoSy by observing a lower "spray score" at the fimbrial end. In a large systematic review with meta-analysis [11] no benefit was found of commercially available contrast media over saline and Doppler sonography was associated with a greater sensitivity and specificity.

However, hyperechogenic contrast media such as Echovist $^{\circledR}$ (Schering AG, Berlin) and SonoVue ${ }^{\circledR}$ (Bracco, Milan) are either no longer available or not licensed for tubal patency testing. Hysterosalpingo-Foam Sonography (HyFoSy) was developed as an alternative for contrast HyCoSy and was introduced in 2010 [12] as a first-line office tubal patency test. Foam is used in the HyFoSy technique to visualize the Fallopian tubes and is created by rigorously mixing $5 \mathrm{ml}$ ExEm $^{\circledR}$-gel (containing hydroxyethyl cellulose and glycerol, IQ Medical Ventures BV, Rotterdam, The Netherlands) with 5 $\mathrm{ml}$ purified $\mathrm{ExEm}^{\circledR}{ }^{\circledR}$-water. The $\mathrm{ExEm}^{\circledR}{ }^{\circledR}$-foam, with a viscosity of $270 \mathrm{cP}$ and containing $94.12 \%$ water, is sufficiently fluid to pass the Fallopian tubes and in the meantime sufficiently stable to show echogenicity for at least five minutes, which is an advantage over saline. In a review on safety aspects and side effects of ExEm-gel [13] it was considered to be appropriate and safe for tubal patency testing. This was confirmed in a recently published retrospective study [14] among 155 women undergoing HyFoSy, reporting no side effects at the follow-up appointment.

This review on tubal patency testing is intended as an update of the literature focusing on clinical aspects of HyFoSy in subfertility workup, like diagnostic accuracy and side effects, 3D and Doppler flow techniques, discomfort and pain, intravasation, pelvic inflammatory disease prevention, and enhancing the chance of pregnancy.

\section{Methods}

A literature search was conducted using PubMed and Embase from the introduction of HyFoSy to 12 March 2019. We searched with terms related to the index test HyFoSy and did not use any filter to maximize the sensitivity of the search. Studies on the use of HyFoSy to confirm occlusion after tubal sterilization were excluded.

Unfortunately, a meta-analysis was not possible due to a too small number of studies, being mutually incomparable for the various subjects of clinical aspects, even for the reliability as a test for tubal patency. We therefore decided to provide a description of published findings and facts as an update of the literature on this subject. The results are summarized in a table containing references per subject, type of the study, study design, and main results (Table 1).

\section{Diagnostic Accuracy and Side Effects}

The first report on efficacy of HyFoSy [15] was a prospective observational cohort study in 73 subfertile women undergoing a HyFoSy. A successful procedure was performed in 67 (92\%) of these 73 women. In 57 women (78\%) tubal patency was observed and no further examination was needed. In 5 women $(7 \%)$ tubal occlusion was confirmed by HSG and in another 5 women (7\%) there was a discrepancy between HSG and HyFoSy findings. Five patients experienced vasovagal discomfort during or after the procedure that resolved spontaneously in time. No serious adverse effects occurred. In the second observational cohort study [16], comparing the results of $20 \mathrm{HyFoSy}$ procedures with LSC, there was a 100\% agreement between HyFoSy and LSC.

In a randomized controlled selective crossover trial ( $n=40)$ comparing HyFoSy with saline HyCoSy [17], the proportion of Fallopian tubes that were classified as patent was higher in the HyFoSy group compared to saline HyCoSy (70,0\% versus 40,0\%, $\mathrm{p}=0.01)$. On crossover testing HyFoSy also performed better than saline HyCoSy. No major postprocedural complications were observed.

In a randomized controlled study in 37 infertile women scheduled for LSC [18], the results of HyFoSy and saline HyCoSy were compared with LSC findings. Tubal patency was in the HyFoSy group concordant with LSC in $94,4 \%$ of cases (sensitivity $87,5 \%$ and specificity $100 \%$ ) compared to $57,8 \%$ in the saline HyCoSy group (sensitivity $50 \%$ and specificity 66,6\%). In a prospective observational study [19] in 132 women HyFoSy and HyFoSy with High Definition Flow (HDF) Doppler technique was compared to saline HyCoSy and LSC as gold standard. Saline HyCoSy and HyFoSy were in comparison to LSC both significantly less accurate $(84.2 \%$; $\mathrm{p}<0.01$, respectively, 92.1\%; $\mathrm{p}<0.01$ ) whereas HyFoSy with HDF Doppler did not significantly differ from LSC (95,8\%; $\mathrm{p}<0.07)$. Although HyFoSy with and without HDF Doppler technique performed better, the authors conclude that saline HyCoSy may be used as an initial test because of its high negative predictive value on tubal occlusion (99.6\% versus 99.5\%, respectively; 99\% for HyFoSy without HDF Doppler technique).

It is good to realize that it is easier to diagnose tubal patency than tubal occlusion due to a difficult differentiation between true and false occlusion caused by, for example, a mucus plug or a spasm [20]. Actually there is no diagnostic test for tubal occlusion, only for tubal patency.

\section{3-Dimensional Ultrasound and Doppler Flow Techniques}

HyCoSy with saline (and air) is, in comparison to HyFoSy, more observer-dependent due to the fact that the hyperechoic characteristics are usually lost within a short time and the inability to examine the whole course of the Fallopian tube in one scanning plane [7]. In attempts to overcome these problems 3-dimensional ultrasound (3D-US) and Doppler techniques [21] were introduced. As the hyperechoic characteristics of HyFoSy are much more stable, there is more time for routine $2 \mathrm{D}$ ultrasound. It is therefore questionable whether these techniques are of additional value for HyFoSy.

In a prospective observational study [22] in 132 subfertile women, all women underwent HyFoSy with new automated $3 \mathrm{D}$ coded contrast imaging (CCI) software (GE Healthcare) with two foam injections followed by $2 \mathrm{D}$ real-time HyFoSy. Because the ExEm-foam was too viscous to pass through the 5 French HyFoSy balloon catheter, the investigators prepared 


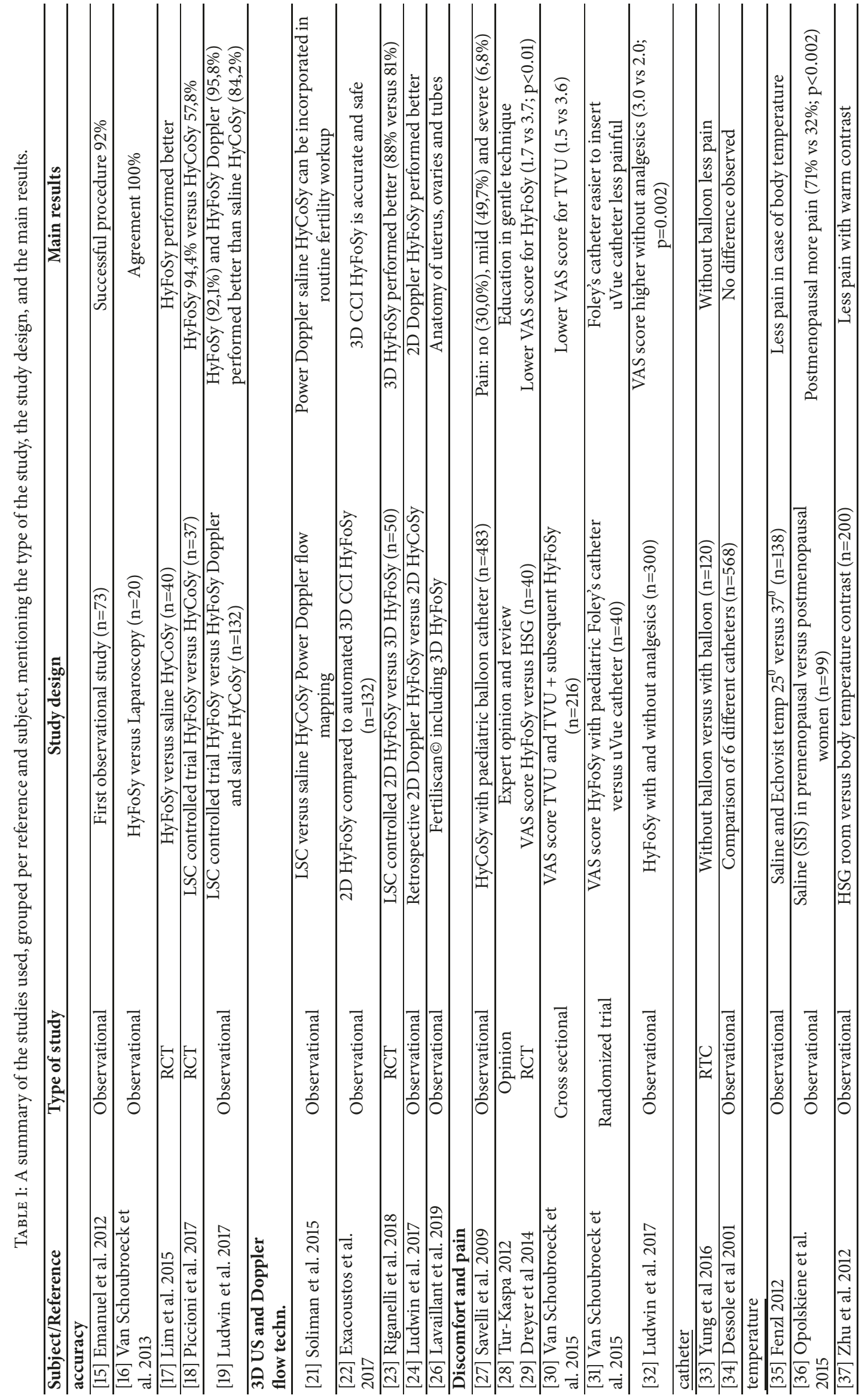




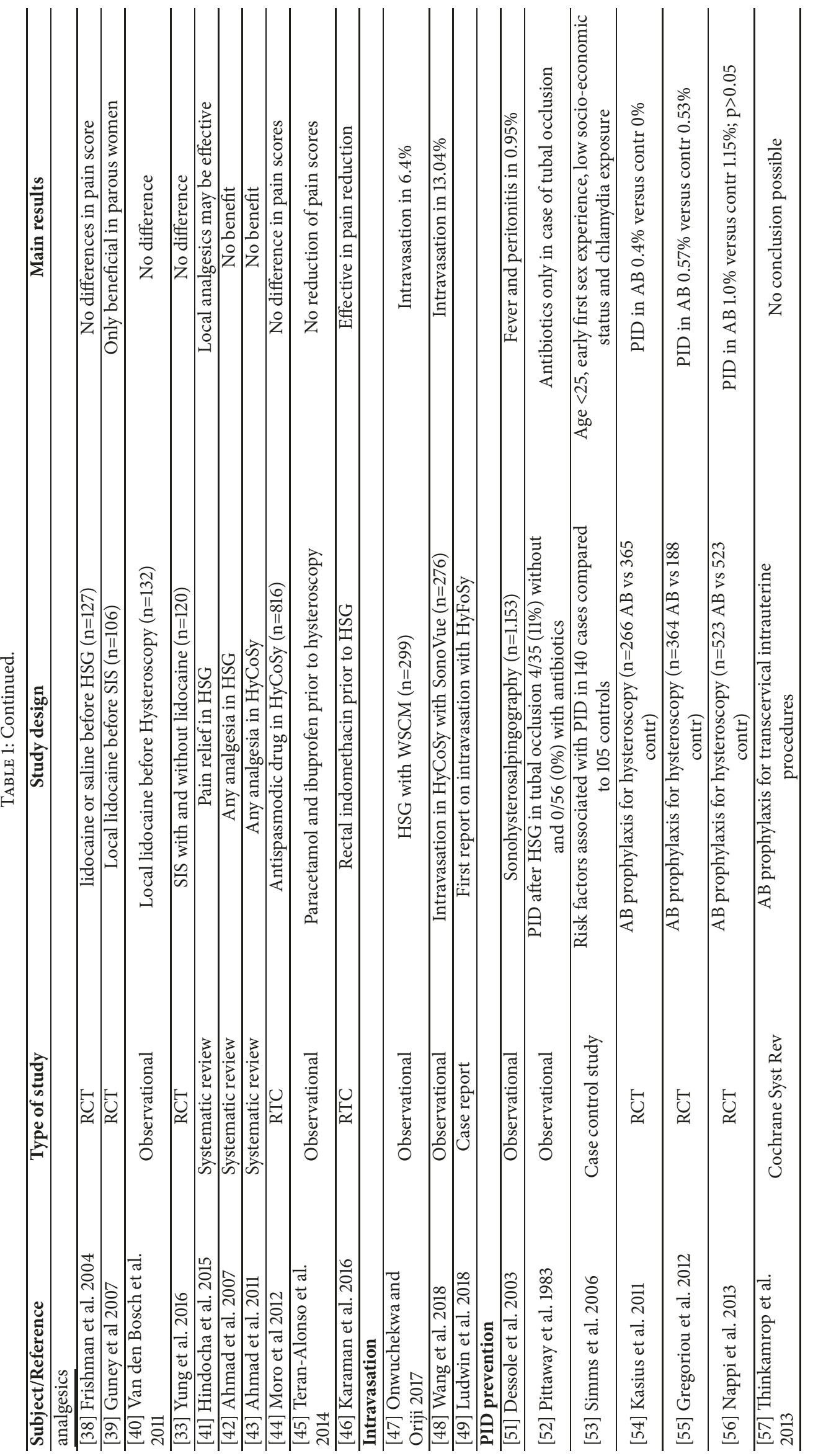




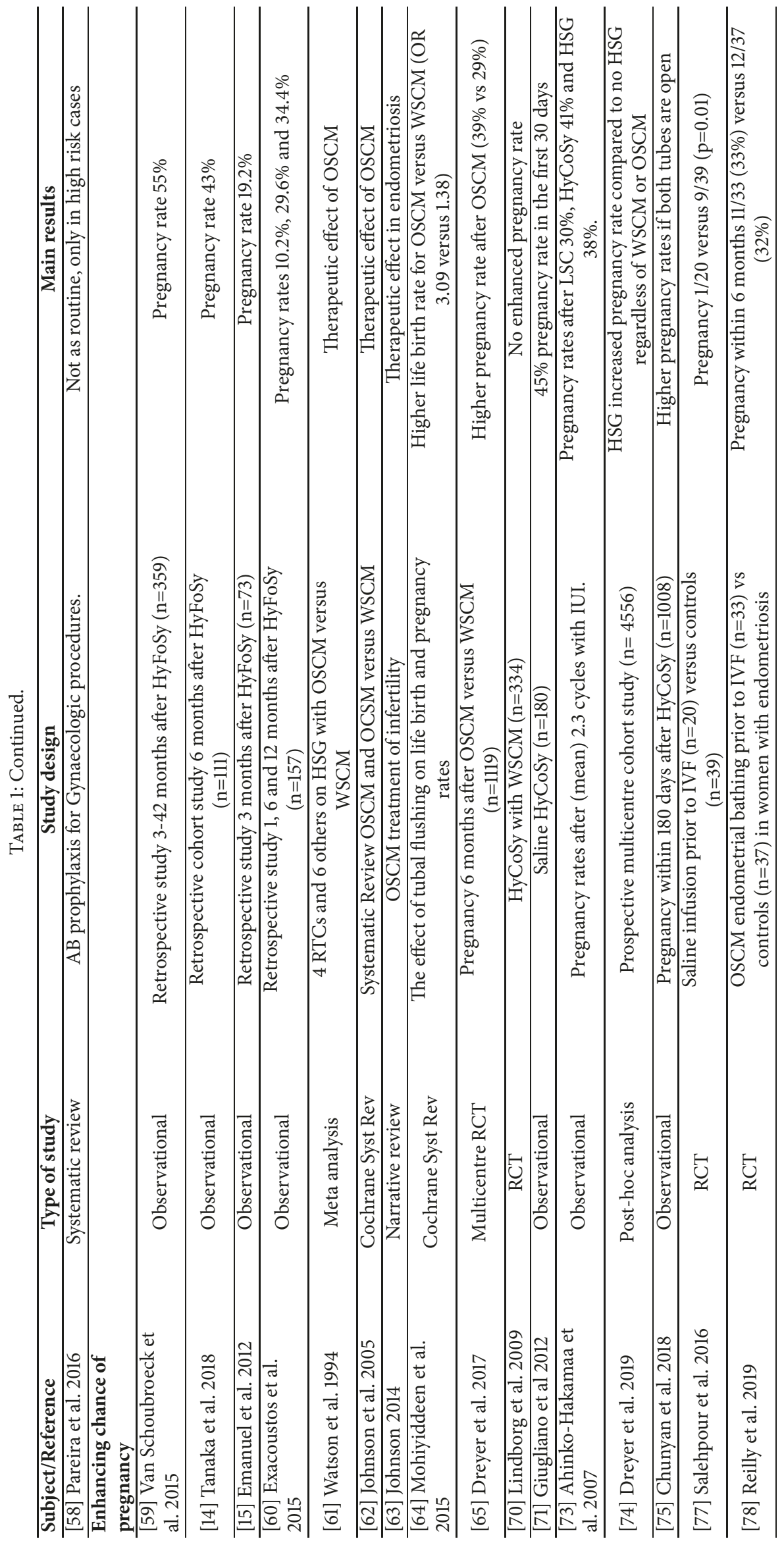




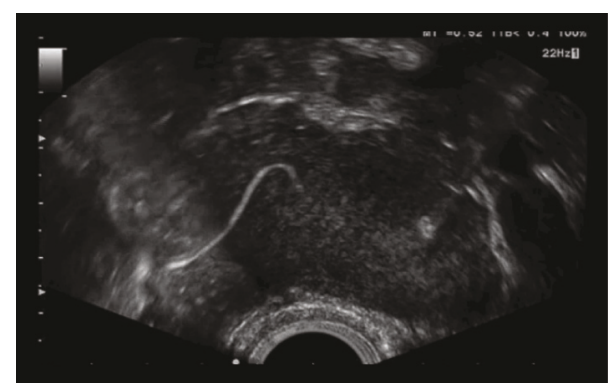

Figure 1: An example of 2D HyFoSy. The clear white line is sufficient evidence for an open Fallopian tube.

a different and unusual off-label ExEm-gel dilution. The concordance rate between the first and second $3 \mathrm{D}$ volume reconstruction and the final $2 \mathrm{D}$ real-time evaluation was $84.8 \%$ and $97.0 \%$, respectively. Vasovagal reactions were observed in two patients and no other adverse effects were noted.

Riganelli et al. [23] described in a pilot study the results of a randomized controlled trial in 50 women who were previously subjected to LSC and randomly assigned to $2 \mathrm{D}-\mathrm{HyF}$ FoSy or $3 \mathrm{D}-\mathrm{HyF}$ FoSy. The $2 \mathrm{D}-\mathrm{HyF}$-Sy was in $81 \%$ of the cases concordant with the LSC, with a sensitivity of $80 \%$ and a specificity of $92 \%$. The $3 \mathrm{D}-\mathrm{HyF}$ FoSy was in $88 \%$ concordant with LSC, with a sensitivity of $98 \%$ and a specificity of $91.4 \%$. Statistical analysis of this difference is lacking in this study. The 3D-HyFoSy procedures were found to be less painful and faster $(\mathrm{P}<0.001)$. In the discussion the authors state that $3 \mathrm{D}-\mathrm{HyFoSy}$ is less operatordependent and more reproducible and it allows postprocedural reconstruction of images. They conclude that, in low risk patients, if the tubes appear obstructed in 2D-HyFoSy, 3D-HyFoSy seems advisable before submitting patients to LSC.

Ludwin et al. [24] concluded from their retrospective study in 50 women that the interobserver reliability and agreement on the diagnosis of tubal patency using stored videos improved when HyFoSy combined with power Doppler technique was used in comparison to 2D-HyCoSy. The relatively small number of patients in this retrospective study and the lack of real-time $2 \mathrm{D}$ information are limitations.

With or without using Doppler techniques, 3D-HyFoSy does not seem to offer benefits above real-time 2D-HyFoSy performed by an ultrasonographer who is familiar with pelvic anatomy (Figure 1). The clear white line indicating foam passage through the proximal part of the tube is sufficient evidence for the absence of a distal occlusion as is known from studies using hysteroscopy and air bubbles [25]. Anyway, from the nine clinical studies [15-24], it can be concluded that HyFoSy appears to be accurate and well-tolerated firstline diagnostic procedure and the $3 \mathrm{D}-\mathrm{HyFoSy}$ technique is helpful for a less experienced operator. Furthermore, 3D scanning offers standardization of pelvic scanning and its use is superb for educational purposes. Recently a novel concept named Fertilityscan $\odot$ was described [26] using 3D-HyFoSy as a women-friendly and cheap assessment for both anatomy and function of the uterus, ovaries, and tubes.

\section{Discomfort and Pain}

Intrauterine application of contrast media may cause discomfort and pain. This may be due to the dilatation of the cervix, application of a catheter, filling of the cavity under pressure, the composition of the contrast medium, or a combination of factors. In an observational study [27] on 483 patients undergoing saline HyCoSy via a paediatric balloon catheter no pain was observed in $30.0 \%$, mild pain in $49.7 \%$, moderate pain in $13.5 \%$, and severe pain in $6.8 \%$ and vasovagal reactions were seen in $4.9 \%$.

In general, a gynaecological examination is for the majority of patients embarrassing and stressful, enhanced by fear or pain [28]. It is important to explain the procedure before starting the examination, to insert the speculum slowly, and, most importantly, to inject the medium very slowly to avoid high intrauterine pressure.

Five studies have been published regarding pain experience during HyFoSy. The first [29] was a randomized controlled trial including 40 women, comparing visual analogue scale (VAS) pain score during tubal patency testing using HyFoSy and serial HSG. For HyFoSy, a small cervical balloonless catheter was placed. For HSG, a hysterophore with one tenaculum on the anterior cervical lip was used. All media were injected in the uterine cavity with the use of an electrical pump with a standardized flow and pressure. This trial showed a lower VAS score in the HyFoSy group compared to the HSG group (median VAS score $1.7 \mathrm{~cm}$; interquartile range (IQR) 4.2 versus median VAS score $3.7 \mathrm{~cm}$; IQR 3.0; $\mathrm{P}<0.01$ ).

In a cross-sectional study [30] on 216 patients the median VAS score for pain during transvaginal ultrasound (TVU) and subsequent HyFoSy was $1.5 \mathrm{~cm}$ (95\% CI, 1.2-1.7) and $3.6 \mathrm{~cm}$ (95\% CI, 3.0-4.0), respectively. To instil the foam, a balloon catheter was placed. One in three women reported the same level of discomfort or pain during TVU as for HyFoSy; $48 \%$ of the women reported the HyFoSy to be neutral/unpleasant, but not painful. There was an inverse association between both patient's age and parity and experienced pain [30].

In a randomized controlled trial [31] including 40 women, HyFoSy was performed using two different balloon catheters, uVue $\mathrm{HSG}^{\otimes}$ catheter or a paediatric Foley's catheter. It was concluded that a paediatric Foley catheter was easier to insert and the HyFoSy was less painful compared to HyFoSy using uVue $\mathrm{HSG}^{\circledR}$ catheter.

One study has been published [32] concerning the use of analgesics during 2D/3D Doppler HyFoSy. In a prospective observational study 300 women were subjected to uterine cavity and tubal patency ultrasound assessment and asked to report VAS pain scores. From October 2012 until March 2013 the procedure was performed without any analgesics in 125 women. From March until the end of the study 175 women received approximately 1 hour before the procedure a tablet containing paracetamol $500 \mathrm{mg}$ and codeine phosphate $30 \mathrm{mg}$. 
During 2D/3D-Doppler-HyFoSy, the median VAS pain score was significantly higher for women not using analgesics (median VAS score $2.0 \mathrm{~cm}$; IQR 1.0-3.0 versus a median VAS score $3.0 \mathrm{~cm}$; IQR 1.3-4.0; $\mathrm{p}=0.002$ ).

There are no other studies concerning pain and the placement of the catheter, filling of the uterus, and local or systemic anaesthesia during HyFoSy. We therefore will report on these aspects in relation to gel instillation sonography (GIS), saline infusing sonography (SIS), HSG, and HyCoSy.

5.1. The Use of Catheter. It has been suggested [33] that SIS performed with an infant feeding tube without a balloon is associated with very low pain levels in comparison to catheters with a balloon (median pain score 10, on a scale of $0-100)$. That is consistent with the theory that most sensory receptors are located in the area of the cervical internal ostium. On the other hand, Dessole et al. [34] compared six different catheters, used in 568 sonohysterograms. The diameter of the catheters varied from Charrier 5 to Charrier 8 , five had a balloon at the tip with a capacity of 3-5mL, and one was equipped with a movable stopper, which is fixed to the external os of the uterus. The authors did not find significant differences with regard to reliability, the physician's ease of use, the insertion time, the volume of contrast medium, and pain.

5.2. Type and Temperature of Contrast. The influence of the contrast medium (Echovist ${ }^{\circledR}$ versus saline) and the temperature $\left(25^{\circ} \mathrm{C}\right.$ versus $\left.37^{\circ} \mathrm{C}\right)$ was investigated in a prospective randomized HyCoSy study [35] including 138 patients. Echovist ${ }^{\circledR}$ induced significantly less pain in comparison to saline at the same temperature $(\mathrm{p}=0.002$ and $\mathrm{p}=0.001)$. Between the two groups there was also a significant difference in pain during the introduction of the same contrast at different temperature $(\mathrm{p}<0.001)$. The most tolerable one for the patient is body temperature. This applies for both contrast media. These results may indicate that Echovist ${ }^{\circledR}$ is more "patient-friendly" than saline. In another study [36] on pain experience during SIS $(n=99)$ it was observed that postmenopausal women experienced pain more often than premenopausal women (71\% versus $32 \%$; $<0.002)$. Also the character of the pain in relation to saline was different: postmenopausal women more often felt a sharp pain (42\%), whereas premenopausal women more often felt gnawing and/or cramping pain (21\%). This may be related to the thin atrophic endometrium in postmenopausal women. Furthermore, in a randomized study on 200 HSG procedures [37] it was observed that warming the contrast to body temperature is associated with less pain and fewer vasovagal episodes.

5.3. Analgesics. In a randomized double-blinded placebocontrolled trial [38] 127 women received $3 \mathrm{~mL} 2 \%$ lidocaine solution or $0.9 \%$ normal saline before undergoing a HSG. As there were no differences with respect to pain scores between both groups, intrauterine lidocaine did not appear to be effective. In a smaller study of 106 women [39], a beneficial effect of intrauterine lidocaine was only seen in parous women undergoing SIS. In a study on 132 patients
[40] undergoing GIS with and without lidocaine containing gel prior to a hysteroscopy no differences were seen either. From another randomized double-blind placebo-controlled trial [33] on 120 patients undergoing SIS it could be concluded that topical or local intrauterine application of lidocaine was not effective in reducing pain.

From a systematic review on pain relief in HSG [41] it was concluded that topical analgesics applied before the procedure may be effective, although the available evidence was of low quality. Also, intravenous opioids may be effective though this must be weighed against their side effects. In other systematic reviews and meta-analysis $[42,43]$ it was concluded that there is no evidence of significant benefit in using any analgesia before HyCoSy or HSG compared to placebo.

Mechanical distension of the uterine walls may cause a release of prostaglandins, resulting in uterine cramps. However in a large randomized double-blind clinical trial [44] $(n=816)$ there was no difference in pain scores between a group receiving an antispasmodic drug (hyoscine-Nbutylbromide) during HyCoSy or a placebo. In another study [45] administration of $1000 \mathrm{mg}$ paracetamol and $600 \mathrm{mg}$ ibuprofen one hour prior to office hysteroscopy did not reduce pain scores. Rectal indomethacin, however, reduced the pain significantly during HSG in a randomized placebocontrolled trial [46].

With regard to discomfort and pain it can be concluded from all available literature that HyFoSy is a well-tolerated procedure, less painful than HSG. There is no role for local and general analgesia. Prophylactic analgesia is not necessary and, in case it is nevertheless considered, rectal application of Indomethacin or codeine tablets seems to be effective.

\section{Intravasation}

Venous intravasation is a well-known complication of HSG, occurring in about $6.4 \%$ of cases [47]. Rarely cerebral and pulmonary oil embolism after oil-soluble contrast media (OSCM), like Lipiodol ${ }^{\circledR}$, has been described in case reports and after water-soluble contrast media WSCM complications like fever, infection, and pain have been described. In a study on HyCoSy with SonVue ${ }^{\circledR}$ intravasation occurred in $13.04 \%$ of 276 patients [48]. The incidence of intravasation was high in case of thin endometrium and high pressure and low on days 5-7 after ending of the menstrual period. Recently a first case on intravasation during HyFoSy has been published [49]. As hydroxyethyl cellulose and glycerol are safe, even in case of intravenous application [13], no clinical signs or complications occurred.

\section{Pelvic Inflammatory Disease Prevention}

As described in the first paragraph, no major postprocedural complications after HyFoSy were observed in nine observational studies [15-24]. Glycerol, one of the components of ExEm-gel, is known to have antimicrobial and virucidal effects [50]. Fever and peritonitis occurred in only $0.95 \%$ of 1.153 patients undergoing sonohysterosalpingography [51]. 
From HSG studies [52] it is known that only patients with an existing hydrosalpinx are at risk of PID and may benefit from prophylactic antibiotics. Age under 25 years, first sex at an early age, lower socioeconomic status, and exposure to chlamydia trachomatis are risk factors for PID [53].

Routine antibiotic prophylaxis is not beneficial and is not recommended in diagnostic hysteroscopy, because of the very low risk of infection [54-56]. In a Cochrane Review on antibiotics for transcervical intrauterine procedures [57] it was concluded that no trials were eligible for inclusion and it is therefore not possible to draw any conclusion. In a large systematic review on antibiotic prophylaxis for gynaecological procedures prior to and during the utilization of assisted reproductive technologies [58] it is also concluded that routine antibiotic prophylaxis is generally not recommended for these procedures. However, patients at risk of pelvic infections should be screened and treated prior to procedures such as HSG, SIS, HyCoSy, HyFoSy, hysteroscopy, embryo transfer, and chromopertubation.

\section{Enhancing the Chance of Pregnancy?}

Up until now, only observational studies are available concerning the chance of subsequent pregnancy after HyFoSy. A retrospective study [59] reported on a 55\% pregnancy rate in 359 women after HyFoSy during a variable followup period of 3 to 42 months. In this study the number of pregnancies was the highest in the cycle of the HyFoSy and the first two cycles after the procedure. In a retrospective cohort study [14], among 111 subfertile women, 48 (43.2\%) women conceived within 6 months after HyFoSy, of whom 24 women conceived naturally. Emanuel et al. [15] reported a natural conception rate of $19.2 \%$ with a median of 3 months after the HyFoSy procedure. In a retrospective observational study [60] regarding 294 subfertile women who underwent HyFoSy, 157 women provided information by phone on their fertility after 12 months. The authors observed a cumulative spontaneous pregnancy rate of $10.2 \%$ within 1 month after HyFoSy, 29.9\% within 6 months, and 34.4\% within 12 months.

More is known about the fertility enhancing effect of tubal flushing at HSG [61]. Tubal flushing with (OSCM) is increasing the odds of pregnancy and live birth in comparison to no intervention or WSCM [62]. It is uncertain whether this is a "tubal flushing" phenomenon, an effect on the intraperitoneal environment, or an implantation enhancing effect on the endometrium. Flushing with an OSCM has been proven to be effective in endometriosis-related infertility [63]. In a recent Cochrane Review [64] the efficacy of tubal flushing with OSCM or WSCM was evaluated. In comparison to no intervention the OSCM group had a higher rate of life birth (OR 3.09, 95\% CI 1,39-6.91) compared to the WSCM group (OR 1.13, 95\% CI 0.67-1.91). Recently a multicentre RCT on 1119 patients [65] showed significantly more ongoing pregnancies in the first 6 months following HSG with OSCM as compared to HSG with WSCM (39\% versus $29 \%$, RR 1.38; $95 \% \mathrm{CI}, 1.17$ to $1.64 ; \mathrm{P}<0.001)$. The increased number of pregnancies in this study was found to be based on pain experienced during the procedure [66]. The use of OSCM in HSG procedures is associated with the occurrence of peritoneal granulomata [67], neonatal hypothyroidism [68], and immunological effects [69].

The clinical impression of enhanced pregnancy rates after HyCoSy with WSCM (Echovist ${ }^{\circledR}$ ) could not be confirmed in a prospective randomized study [70] $(n=334)$. In an observational study on 180 patients after saline HyCoSy [71] a possible beneficial effect of HyCoSy was observed directly after the procedure. The pregnancy rate was significantly higher in the first 30 days after HyCoSy (45\%) compared to other months of observation after HyCoSy $(\mathrm{p}<0.0005)$. One has to keep in mind however that this is comparable to a $42 \%$ natural conception rate observed [72] in the first cycle of the normal population. In a retrospective study [73] on 559 patients treated with intrauterine insemination (IUI) it was observed that the cumulative pregnancy rates (mean 2,3 cycles) after LSC, saline HyCoSy, and HSG were $30 \%, 41 \%$, and $38 \%$, respectively. In a recent post hoc analysis of a prospective multicentre cohort study [74] among 4556 couples with unexplained infertility HSG increased the ongoing pregnancy rate compared to no HSG (adjusted hazard ratio $1.40,95 \%$ CI 1.16-1.70) regardless of WSCM or OSCM was used. Furthermore, in a large study on 1008 infertile patients patency of both Fallopian tubes and the absence of injective resistance turned out to be independent factors associated with the ability to conceive after HyCoSy [75]. This is consistent with the one-half reduction of clinical pregnancies in a large study on the effect of unilateral tubal abnormalities on the results of intrauterine inseminations [76].

In a prospective randomized controlled trial on intrauterine saline infusion as a form of pregnancy enhancing endometrial injury during IVF cycles in 63 patients with recurrent implantation failure [77] a possible negative effect of saline on reproductive outcomes was observed. In this study a clinical pregnancy occurred in 1 out of the 20 women undergoing intrauterine saline infusion on days 35 in the stimulation phase as opposed to 9 out of 43 women without an infusion $(\mathrm{p}<0.05)$. In a randomized study [78] comparing uterine bathing with OSCM prior to IVF with IVF alone, no evidence was found of any beneficial effect.

ExEm-foam is safe and even passed the mouse-embryotest [13]. Although in observational studies we could not find any negative effect on fertility after the HyFoSy procedure, we have to conclude that no studies on postprocedure pregnancy rates are available comparing HyFoSy with other or no intervention.

\section{Conclusion and Discussion}

Although most of the studies presented in this review are small with an observational design, it can be concluded that HyFoSy is a promising alternative for HSG with regard to accuracy and effectiveness. HyFoSy lacks radiation and iodine exposure, which is a benefit in comparison to HSG. With or without using Doppler techniques, 3D-HyFoSy 
does not seem to offer benefits above real-time 2D-HyFoSy. However, 3D scanning offers standardization of pelvic scanning and may be performed by a less experienced operator. HyFoSy is a well-tolerated and less painful procedure than HSG without a need for the use of analgesics. No serious or severe complications have been reported after more than 350.000 procedures. Routine antibiotic prophylaxis is generally not recommended; however patients at risk at pelvic infections should be screened and treated accordingly prior to the HyFoSy procedure. Moreover, there appears to be no detrimental effect of HyFoSy on fertility and there might even be a beneficial effect in the first three menstrual cycles after the procedure on enhancing pregnancies.

\section{Future Perspectives}

As HyFoSy is still a relatively new tubal patency test, only observational data are available. Therefore, robust randomized controlled trials are needed to draw firm conclusion on the degree of accuracy and effectiveness of HyFoSy and the fertility enhancing effect. As ExEm-foam is not yet FDA-approved, large trials and clinical use of HyFoSy in the US are lacking. In the Netherlands a large randomized controlled study, the so-called FOAM trial, comparing the effectiveness and costs of HyFoSy with HSG, is currently ongoing [79]. In this study subfertile patients ( $\mathrm{N}=1163)$, who are scheduled for tubal patency testing during their fertility workup, undergo both HSG and HyFoSy in a random order. If the results of both tubal tests are discordant, women will be randomly allocated to either a management strategy based on HyFoSy or a management strategy based on HSG, implicating either a LSC or a strategy that assumes tubal patency. The primary outcome of this trial is an ongoing pregnancy leading to live birth within 12 months after randomization. Recruitment for this trial is expected to be completed in the fall of 2018. Therefore, the results will be available around 2020.

With regard to the fertility enhancing effect randomized controlled trials comparing HyFoSy with other or no intervention are urgently needed.

\section{Disclosure}

This research was not funded or financially supported by any grant; that is, no external funding was requested or provided for this study.

\section{Conflicts of Interest}

Niek Exalto and Mark Hans Emanuel are the inventors of GIS and $\mathrm{HyFoSy}$ with $\mathrm{ExEm}^{\circledR}{ }^{\circledR}$ gel. They are shareholders in Giskit B. V., the manufacturer of GIS $^{\circledR}$-Kit and ExEm ${ }^{\circledR}$-Foam Kit, and receive royalties from this company. This survey of the clinical aspects of this new technique is a consequence of their involvement.

\section{Acknowledgments}

We are very grateful to Joukje van Rijswijk, Dr. Velja Mijatovic, and Prof. Dr. Nils Lambalk for their critical contributions from the first manuscript onwards, their additions, and suggestions for improvements.

\section{References}

[1] National institute for Health and Care Excellence (NICE), "Fertility problems: assessment and treatment," 2013, https://www .nice.org.uk/guidance/CG156.

[2] J. A. Steinkeler, C. A. Woodfield, E. Lazarus, and M. M. Hillstrom, "Female infertility: a systematic approach to radiologic imaging and diagnosis," RadioGraphics, vol. 29, no. 5, pp. 1353$1370,2009$.

[3] C. P. Lim, Z. Hasafa, S. Bhattacharya, and A. Maheshwari, "Should a hysterosalpingogram be a first-line investigation to diagnose female tubal subfertility in the modern subfertility workup?" Human Reproduction, vol. 26, no. 5, pp. 967-971, 2011.

[4] U. Deichert, R. Schlief, M. Van De Sandt, and I. Juhnke, "Transvaginal hysterosalpingo-contrast-sonography (Hy-CoSy) compared with conventional tubal diagnostics," Human Reproduction, vol. 4, no. 4, pp. 418-424, 1989.

[5] F. Mitri, A. D. Andronikou, and S. Perpinial, "A clinical comparison of sonographic hydropertubation and hysterosalpingography," International Journal of Obstetrics \& Gynaecology, vol. 48, pp. 1031-1036, 1991.

[6] R. D. Saunders, J. M. Shwayder, and S. T. Nakajima, "Current methods of tubal patency assessment," Fertility and Sterility, vol. 95, no. 7, pp. 2171-2179, 2011.

[7] D. E. Luciano, C. Exacoustos, and A. A. Luciano, "Contrast ultrasonography for tubal patency," Journal of Minimally Invasive Gynecology, vol. 21, no. 6, pp. 994-998, 2014.

[8] G. Lo Monte, G. Capobianco, I. Piva, D. Caserta, S. Dessole, and R. Marci, "Hysterosalpingo contrast sonography (HyCoSy): let's make the point!", Archives of Gynecology and Obstetrics, vol. 291, no. 1, pp. 19-30, 2015.

[9] Y. Wang and L. Qian, "Three- or four-dimensional hysterosalpingo contrast sonography for diagnosing tubal patency in infertile females: a systematic review with meta-analysis," British Journal of Radiology, vol. 89, Article ID 20151013, 2016.

[10] Y. He, X. Ma, J. Xu et al., "Comparison of Assessment Methods for Fallopian Tubal Patency and Peritubal Adhesion Between Transvaginal 4-Dimensional Hysterosalpingo-Contrast Sonography and Laparoscopic Chromopertubation," Journal of the American Institute of Ultrasound in Medicine, vol. 36, no. 3, pp. 547-556, 2017.

[11] S. Maheux-Lacroix, A. Boutin, L. Moore et al., "Hysterosalpingosonography for diagnosing tubal occlusion in subfertile women: A systematic review with meta-analysis," Human Reproduction, vol. 29, no. 5, pp. 953-963, 2014.

[12] M. H. Emanuel and N. Exalto, "Hysterosalpingo-foam sonography (HyFoSy): A new technique to visualize tubal patency," Ultrasound in Obstetrics \& Gynecology, vol. 37, no. 4, pp. 498499, 2011.

[13] N. Exalto, M. Stassen, and M. H. Emanuel, "Safety aspects and side-effects of ExEm-gel and foam for uterine cavity distension and tubal patency testing," Reproductive BioMedicine Online, vol. 29, no. 5, pp. 534-540, 2014. 
[14] K. Tanaka, J. Chua, R. Cincotta, E. L. Ballard, and G. Duncombe, "Hysterosalpingo-foam sonography (HyFoSy): Tolerability, safety and the occurrence of pregnancy post-procedure," Australian and New Zealand Journal of Obstetrics and Gynaecology, vol. 58, no. 1, pp. 114-118, 2018.

[15] M. H. Emanuel, M. Van Vliet, M. Weber, and N. Exalto, "First experiences with hysterosalpingo-foam sonography (HyFoSy) for office tubal patency testing," Human Reproduction, vol. 27, no. 1, pp. 114-117, 2012.

[16] D. Van Schoubroeck, T. Van Den Bosch, C. Meuleman, C. Tomassetti, T. D'Hooghe, and D. Timmerman, "The use of a new gel foam for the evaluation of tubal patency," Gynecologic and Obstetric Investigation, vol. 75, no. 3, pp. 152-156, 2013.

[17] S. L. Lim, J. J. Jung, S. L. Yu, and H. Rajesh, "A comparison of hysterosalpingo-foam sonography (HyFoSy) and hysterosalpingo-contrast sonography with saline medium (HyCoSy) in the assessment of tubal patency," European Journal of Obstetrics \& Gynecology and Reproductive Biology, vol. 195, pp. 168-172, 2015.

[18] M. G. Piccioni, L. Riganelli, V. Filippi et al., "Sonohysterosalpingography: comparison of foam and saline solution," Journal of Clinical Ultrasound, vol. 45, no. 2, pp. 67-71, 2017.

[19] I. Ludwin, A. Ludwin, M. Wiechec et al., "Accuracy of hysterosalpingo-foam sonography in comparison to hysterosalpingocontrast sonography with air/saline and to laparoscopy with dye," Human Reproduction, vol. 32, no. 4, pp. 758-769, 2017.

[20] H. Rajesh, S. L. Lim, and S. L. Yu, "Hysterosalpingo-foam sonography: patient selection and perspectives," International Journal of Women's Health, vol. 9, pp. 23-32, 2017.

[21] A. A. Soliman, W. Shaalan, T. Abdel-Dayem et al., "Power doppler flow mapping and four-dimensional ultrasound for evaluating tubal patency compared with laparoscopy," European Journal of Obstetrics \& Gynecology and Reproductive Biology, vol. 195, pp. 83-87, 2015.

[22] C. Exacoustos, A. Pizzo, L. Lazzeri, A. Pietropolli, E. Piccione, and E. Zupi, "Three-dimensional hysterosalpingo contrast sonography with gel foam: methodology and feasibility to obtain 3-dimensional volumes of tubal shape," Journal of Minimally Invasive Gynecology, vol. 24, no. 5, pp. 827-832, 2017.

[23] L. Riganelli, A. Casorelli, J. Caccetta et al., "Ultrasonography reappraisal of tubal patency in assisted reproduction technology patients: Comparison between 2D and 3Dsonohysterosalpingography. a pilot study," Minerva Ginecologica, vol. 70, no. 2, pp. 123-128, 2018.

[24] I. Ludwin, A. Ludwin, C. O. Nastri, M. A. Coelho Neto, J. Kottner, and W. P. Martins, "Inter-rater reliability of Air/Saline HyCoSy, HyFoSy and HyFoSy combined with power doppler for screening tubal patency," Ultraschall in der Medizin/European Journal of Ultrasound (UiM/EJU), 2017.

[25] J. P. Parry, D. Riche, J. Aldred et al., "Proximal tubal patency demonstrated through air infusion during flexible office hysteroscopy is predictive of whole tubal patency," Journal of Minimally Invasive Gynecology, vol. 24, no. 4, pp. 646-652, 2017.

[26] J. Levaillant, M. Pasquier, and N. Massin, "A novel concept for female infertility exploration: the Fertiliscan $\odot$, a dedicated all-in-one 3D ultrasound exploration," Journal of Gynecology Obstetrics and Human Reproduction, vol. 48, no. 5, pp. 363-367, 2019.

[27] L. Savelli, P. Pollastri, M. Guerrini et al., "Tolerability, side effects, and complications of hysterosalpingocontrast sonography (HyCoSy)," Fertility and Sterility, vol. 92, no. 4, pp. 14811486, 2009.
[28] T. Tur-Kaspa, "Fear no pain: uterine cavity and tubal patency assessment tests should be pain free," Ultrasound in Obstetrics \& Gynecology, vol. 39, no. 3, pp. 247-250, 2012.

[29] K. Dreyer, R. Out, P. G. A. Hompes, and V. Mijatovic, "Hysterosalpingo-foam sonography, a less painful procedure for tubal patency testing during fertility workup compared with (serial) hysterosalpingography: a randomized controlled trial," Fertility and Sterility, vol. 102, no. 3, pp. 821-825, 2014.

[30] D. Van Schoubroeck, T. Van Den Bosch, L. Ameye, A. S. Boes, T. D'Hooghe, and D. Timmerman, "Pain during Fallopiantube patency testing by hysterosalpingo-foam sonography," Ultrasound in Obstetrics \& Gynecology, vol. 45, no. 3, pp. 346350, 2015.

[31] D. Van Schoubroeck, T. Van den Bosch, L. Ameye, T. D’Hooghe, and D. Timmerman, "Hysterosalpingo-foam sonography (HyFoSy) using two different balloon catheters: a randomized trial," Journal of Gynecologic Surgery, vol. 12, no. 1, pp. 41-44, 2015.

[32] I. Ludwin, W. P. Martins, C. O. Nastri, and A. Ludwin, "Pain intensity during ultrasound assessment of uterine cavity and tubal patency with and without painkillers: prospective observational study," Journal of Minimally Invasive Gynecology, vol. 24, no. 4, pp. 599-608, 2017.

[33] S. S. F. Yung, S. F. Lai, M. T. Lam et al., "Randomized, controlled, double-blind trial of topical lidocaine gel and intrauterine lidocaine infusion for pain relief during saline contrast sonohysterography," Ultrasound in Obstetrics \& Gynecology, vol. 47, no. 1, pp. 17-21, 2016.

[34] S. Dessole, M. Farina, G. Capobianco, G. B. Nardelli, G. Ambrosini, and G. B. Meloni, "Determining the best catheter for sonohysterography," Fertility and Sterility, vol. 76, no. 3, pp. 605-609, 2001.

[35] V. Fenzl, "Effect of different ultrasound contrast materials and temperatures on patient comfort during intrauterine and tubal assessment for infertility," European Journal of Radiology, vol. 81, no. 12, pp. 4143-4145, 2012.

[36] G. Opolskiene, S. Radzvilaite, D. Bartkeviciene, D. Ramasauskaite, J. Zakareviciene, and G. Drasutiene, "Pain experience during saline-contrast sonohysterography differs between premenopausal and postmenopausal women," Journal of Clinical Ultrasound, vol. 44, no. 5, pp. 267-271, 2016.

[37] Y.-Y. Zhu, Y.-Z. Mao, and W.-L. Wu, "Comparison of warm and cold contrast media for hysterosalpingography: a prospective, randomized study," Fertility and Sterility, vol. 97, no. 6, pp. 14051409, 2012.

[38] G. N. Frishman, P. K. Spencer, S. Weitzen, S. Plosker, and F. Shafi, "The use of intrauterine lidocaine to minimize pain during hysterosalpingography: a randomized trial," Obstetrics \& Gynecology, vol. 103, no. 6, pp. 1261-1266, 2004.

[39] M. Guney, B. Oral, G. Bayhan, and T. Mungan, "Intrauterine lidocaine infusion for pain relief during saline solution infusion sonohysterography: a randomized, controlled trial," Journal of Minimally Invasive Gynecology, vol. 14, no. 3, pp. 304-310, 2007.

[40] T. Van Den Bosch, D. Van Schoubroeck, A. Daemen et al., "Lidocaine does not reduce pain perception during gel instillation sonography or subsequent office hysteroscopy: results of a randomized trial," Gynecologic and Obstetric Investigation, vol. 71, no. 4, pp. 236-239, 2011.

[41] A. Hindocha, L. Beere, H. O’Flynn, A. Watson, and G. Ahmad, "Pain relief in hysterosalpingography," Cochrane Database of Systematic Reviews, vol. 9, Article ID Cd006106, 2015. 
[42] G. Ahmad, J. Duffy, and A. J. Watson, "Pain relief in hysterosalpingography," Cochrane Database of Systematic Reviews, vol. 2, Article ID CD006106, 2007.

[43] G. Ahmad, S. Attarbashi, H. O’Flynn, and A. J. S. Watson, "Pain relief in office gynaecology: A systematic review and meta-analysis," European Journal of Obstetrics \& Gynecology and Reproductive Biology, vol. 155, no. 1, pp. 3-13, 2011.

[44] F. Moro, L. Selvaggi, F. Sagnella et al., "Could antispasmodic drug reduce pain during hysterosalpingo-contrast sonography (HyCoSy) in infertile patients? a randomized double-blind clinical trial," Ultrasound in Obstetrics \& Gynecology, vol. 39, no. 3, pp. 260-265, 2012.

[45] M. J. Teran-Alonso, J. D. Santiago, R. Usandizaga, and I. Zapardiel, "Evaluation of pain in office hysteroscopy with prior analgesic medication: a prospective randomized study," European Journal of Obstetrics \& Gynecology and Reproductive Biology, vol. 178, pp. 123-127, 2014.

[46] E. Karaman, N. Çim, I. Alkiş, A. Yildirim, and R. Yildizhan, "Rectal indomethacin use in pain relief during hysterosalpingography: a randomized placebo controlled trial," Journal of Obstetrics and Gynaecology Research, vol. 42, no. 2, pp. 195-201, 2016.

[47] C. Onwuchekwa and V. Oriji, "Hysterosalpingographic (HSG) pattern of infertility in women of reproductive age," Journal of Human Reproductive Sciences, vol. 10, no. 3, pp. 178-184, 2017.

[48] W. Wang, Q. Zhou, X. Zhou, Z. Chen, and H. Zhang, "Influence factors on contrast agent venous intravasation during transvaginal 4-dimensional hysterosalpingo-contrast sonography," Journal of Ultrasound in Medicine, vol. 37, no. 10, pp. 2379-2385, 2018.

[49] A. Ludwin, I. Ludwin, and W. P. Martins, "Venous intravasation during evaluation of tubal patency by ultrasound contrast imaging," Ultrasound in Obstetrics \& Gynecology, vol. 51, no. 1, pp. 142-146, 2018.

[50] J. W. Fluhr, R. Darlenski, and C. Surber, "Glycerol and the skin: holistic approach to its origin and functions," British Journal of Dermatology, vol. 159, no. 1, pp. 23-34, 2008.

[51] S. Dessole, M. Farina, G. Rubattu, E. Cosmi, G. Ambrosini, and G. B. Nardelli, "Side effects and complications of sonohysterosalpingography," Fertility and Sterility, vol. 80, no. 3, pp. 620-624, 2003.

[52] D. E. Pittaway, A. C. Winfield, W. Maxson, J. Daniell, C. Herbert, and A. C. Wentz, "Prevention of acute pelvic inflammatory disease after hysterosalpingography: efficacy of doxycycline prophylaxis," American Journal of Obstetrics \& Gynecology, vol. 147, no. 6, pp. 623-626, 1983.

[53] I. Simms, J. M. Stephenson, H. Mallinson et al., "Risk factors associated with pelvic inflammatory disease," Sexually Transmitted Infections, vol. 82, no. 6, pp. 452-457, 2006.

[54] J. C. Kasius, F. J. Broekmans, B. C. Fauser, P. Devroey, and H. M. Fatemi, "Antibiotic prophylaxis for hysteroscopy evaluation of the uterine cavity," Fertility and Sterility, vol. 95, no. 2, pp. 792794, 2011.

[55] O. Gregoriou, P. Bakas, C. Grigoriadis, M. Creatsa, C. Sofoudis, and G. Creatsas, "Antibiotic prophylaxis in diagnostic hysteroscopy: is it necessary or not?" European Journal of Obstetrics \& Gynecology and Reproductive Biology, vol. 163, no. 2, pp. 190192, 2012.

[56] L. Nappi, A. Di Spiezio Sardo, M. Spinelli et al., "A multicenter, double-blind, randomized, placebo-controlled study to assess whether antibiotic administration should be recommended during office operative hysteroscopy," Reproductive Sciences, vol. 20, no. 7, pp. 755-761, 2013.

[57] J. Thinkhamrop, M. Laopaiboon, and P. Lumbiganon, "Prophylactic antibiotics for transcervical intrauterine procedures," Cochrane Database of Systematic Reviews, vol. 5, Article ID CD005637, 2013.

[58] N. Pereira, A. P. Hutchinson, J. P. Lekovich, E. Hobeika, and R. T. Elias, "Antibiotic prophylaxis for gynecologic procedures prior to and during the utilization of assisted reproductive technologies: a systematic review," Journal of Pathogens, vol. 2016, Article ID 4698314, 8 pages, 2016.

[59] D. Van Schoubroeck, T. V. den Bosch, K. Van Tornout, T. D'Hooghe, and D. Timmerman, "Spontaneous conception after hysterosalpingo-foam sonography (HyFoSy)," Ultrasound in Obstetrics \& Gynecology, vol. 46, supplement 1, no. S1, pp. 51-51, 2015.

[60] C. Exacoustos, F. Tiberio, B. Szabolcs, V. Romeo, E. Romanini, and E. Zupi, "Can tubal flushing with hysterosalpingo-foam sonography (HyFoSy) media increase women's chances of pregnancy?" Journal of Minimally Invasive Gynecology, vol. 22, no. 6, p. S238, 2015.

[61] A. Watson, P. Vandekerckhove, R. Lilford, A. Vail, I. Brosens, and E. Hughes, "A meta-analysis of the therapeutic role of oil soluble contrast media at hysterosalpingography: a surprising result?" Fertility and Sterility, vol. 61, no. 3, pp. 470-477, 1994.

[62] N. Johnson, P. Vandekerckhove, A. Watson et al., "Tubal flushing for subfertility," Cochrane Database of Systematic Reviews, vol. 2, Article ID CD003718, 2005.

[63] N. P. Johnson, "Review of lipiodol treatment for infertility an innovative treatment for endometriosis-related infertility?" Australian and New Zealand Journal of Obstetrics and Gynaecology, vol. 54, no. 1, pp. 9-12, 2014.

[64] L. Mohiyiddeen, A. Hardiman, C. Fitzgerald et al., "Tubal flushing for subfertility," Cochrane Database of Systematic Reviews, vol. 2015, no. 5, Article ID CD003718, 2015.

[65] K. Dreyer, J.van Rijswijk, V. Mijatovic et al., "Oil-based or waterbased contrast for hysterosalpingography in infertile women," NEJM, vol. 376, pp. 2043-2052, 2017.

[66] N. van Welie, K. Dreyer, J. van Rijswijk et al., "Treatment effect of oil-based contrast at HSG is dependent on pain at HSG but not on volume of contrast," Fertility and Sterility, vol. 110, no. 4, p. e40, 2018.

[67] C. M. Grosskinsky, R. L. Clark, P. A. Wilson, and D. B. Novotny, "Pelvic granulomata mimicking endometriosis following the administration of oil-based contrast media for hysterosalpingography," Obstetrics \& Gynecology, vol. 83, no. 5, pp. 890-892, 1994.

[68] M. Satoh, K. Aso, and Y. Katagiri, "Thyroid dysfunction in neonates born to mothers who have undergone hysterosalpingography involving an oil-soluble iodinated contrast medium," Hormone Research in Paediatrics, vol. 84, no. 6, pp. 370-375, 2015.

[69] G. Izumi, K. Koga, M. Takamura et al., "Oil-soluble contrast medium (OSCM) for hysterosalpingography modulates dendritic cell and regulatory $\mathrm{T}$ cell profiles in the peritoneal cavity: A possible mechanism by which OSCM enhances fertility," The Journal of Immunology, vol. 198, no. 11, pp. 4277-4284, 2017.

[70] L. Lindborg, J. Thorburn, C. Bergh, and A. Strandell, "Influence of HyCoSy on spontaneous pregnancy: A randomized controlled trial," Human Reproduction, vol. 24, no. 5, pp. 1075-1079, 2009. 
[71] E. Giugliano, E. Cagnazzo, E. Bazzan, A. Patella, and R. Marci, "Hysterosalpingo-contrast sonography: Is possible to quantify the therapeutic effect of a diagnostic test?" Clinical and Experimental Reproductive Medicine, vol. 39, no. 4, pp. 161-165, 2012.

[72] D. D. Baird, A. J. Wilcox, and C. R. Weinberg, "Use of time to pregnancy to study environmental exposures," American Journal of Epidemiology, vol. 124, no. 3, pp. 470-480, 1986.

[73] K. Ahinko-Hakamaa, H. Huhtala, and H. Tinkanen, "The validity of air and saline hysterosalpingo-contrast sonography in tubal patency investigation before insemination treatment," European Journal of Obstetrics \& Gynecology and Reproductive Biology, vol. 132, no. 1, pp. 83-87, 2007.

[74] K. Dreyer, R. van Eekelen, R. Tjon-Kon-Fat et al., "The therapeutic effect of hysterosalpingography in couples with unexplained subfertility: a post-hoc analysis of a prospective multi-centre cohort study," Reproductive BioMedicine Online, vol. 38, no. 2, pp. 233-239, 2019.

[75] G. Chunyan, P. Bin, Y. Ping et al., "Assessment of the influence on spontaneous pregnancy of hysterosalpingo-contrast sonography," BioMed Research International, vol. 2018, Article ID 4901281, 8 pages, 2018.

[76] T. Cochet, N. Gatimel, J. Moreau et al., "Effect of unilateral tubal abnormalities on the results of intrauterine inseminations," Reproductive BioMedicine Online, vol. 35, no. 3, pp. 314-317, 2017.

[77] S. Salehpour, M. Zamaniyan, N. Saharkhiz et al., "Does intrauterine saline infusion by intrauterine insemination (IUI) catheter as endometrial injury during IVF cycles improve pregnancy outcomes among patients with recurrent implantation failure?: An RCT,' International Journal of Reproductive BioMedicine, vol. 14, no. 9, pp. 583-588, 2016.

[78] S. J. Reilly, E. J. Glanville, B. Dhorepatil, L. R. Prentice, B. W. Mol, and N. P. Johnson, "The IVF-LUBE trial - a randomized trial to assess Lipiodol ${ }^{\circledR}$ uterine bathing effect in women with endometriosis or repeat implantation failure undergoing IVF," Reproductive BioMedicine Online, vol. 38, no. 3, pp. 380-386, 2019.

[79] J.van Rijswijk, N. van Welie, K. Dreyer et al., "The FOAM study: is Hysterosalpingo foam sonography (HyFoSy) a cost-effective alternative for hysterosalpingography (HSG) in assessing tubal patency in subfertile women? study protocol for a randomized controlled trialfor a randomized controlled trial," BMC Womens Health, vol. 18, no. 64, 2018. 


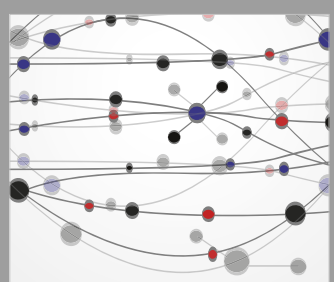

The Scientific World Journal
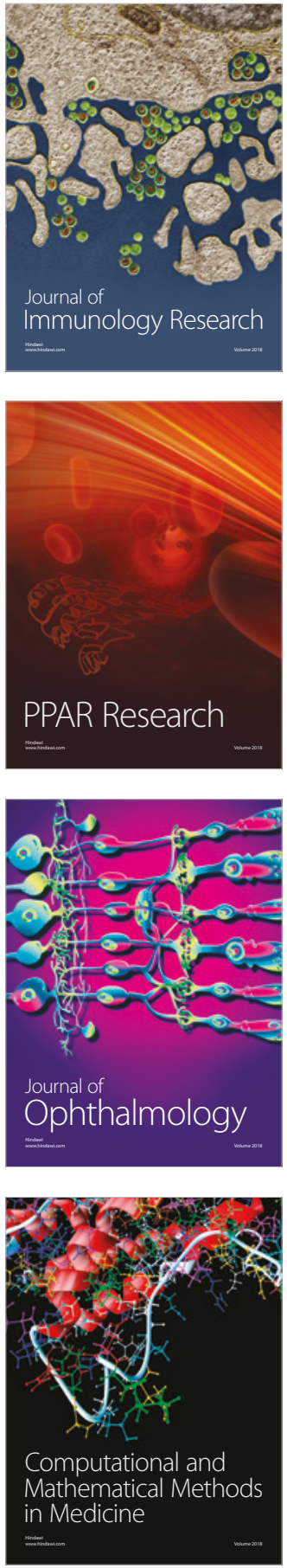

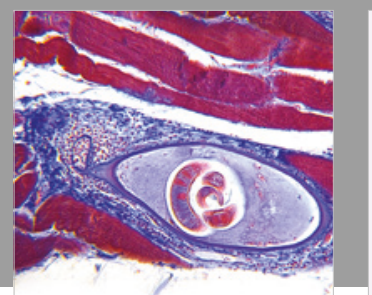

Gastroenterology Research and Practice

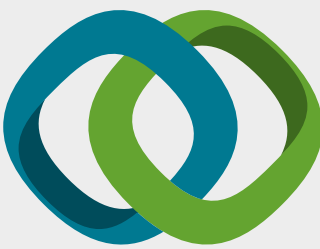

\section{Hindawi}

Submit your manuscripts at

www.hindawi.com
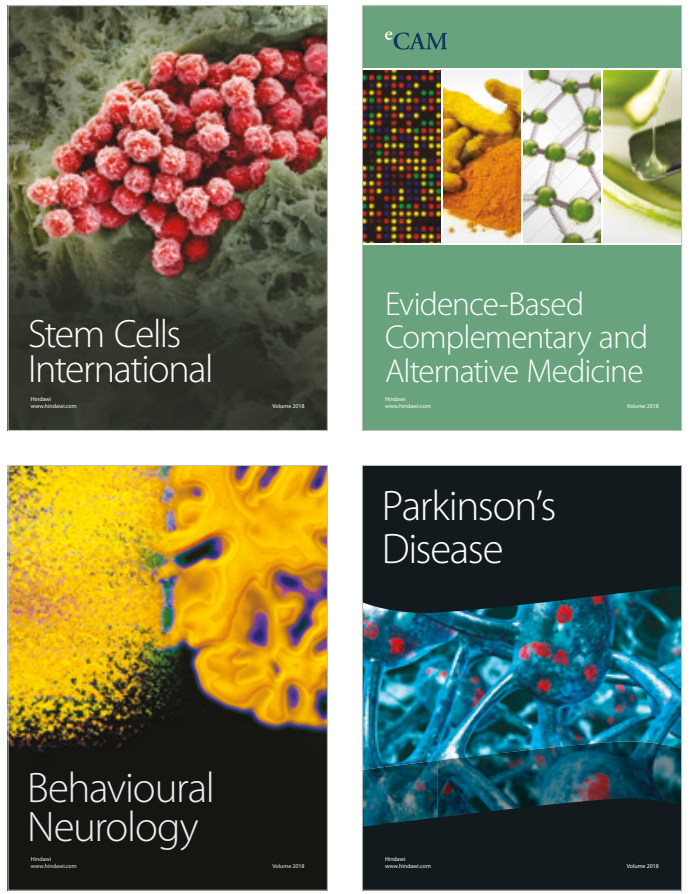

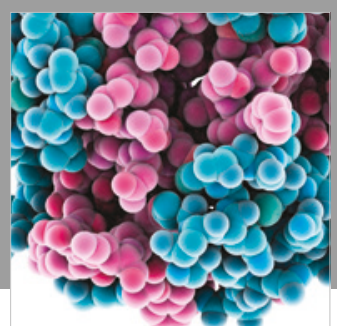

ournal of

Diabetes Research

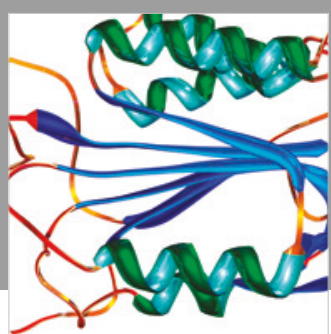

Disease Markers
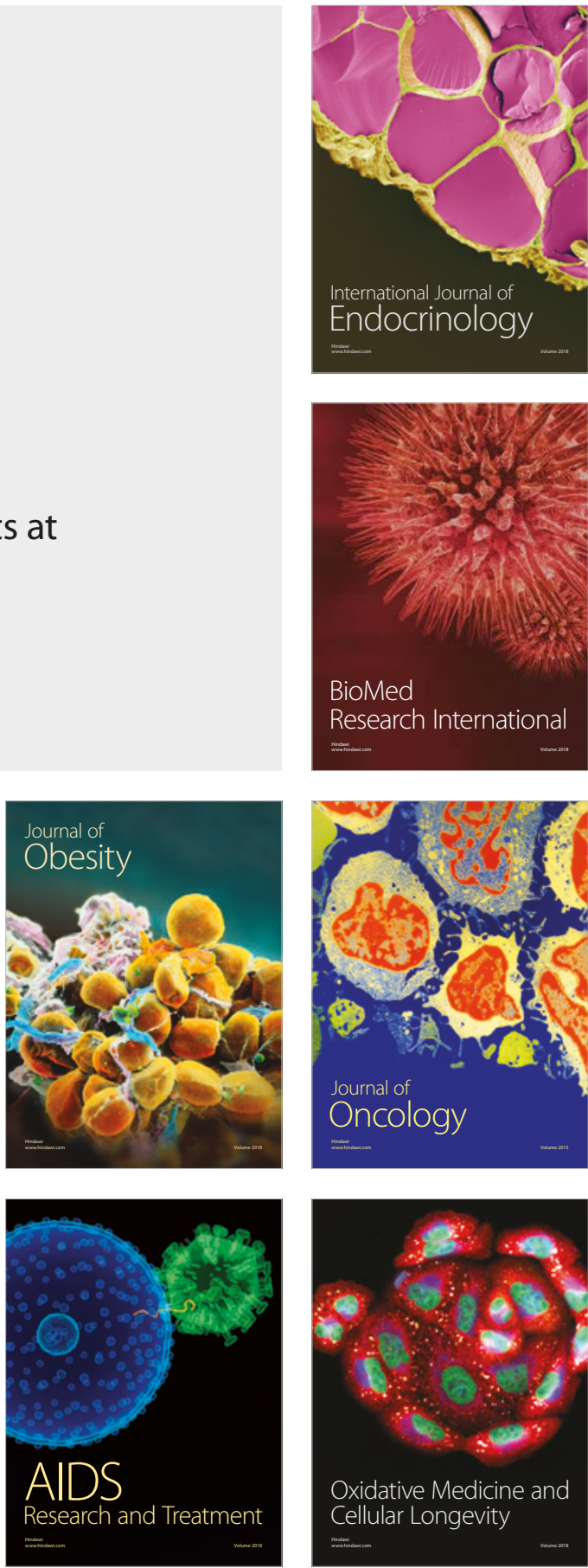